\title{
Toxicogenomic identification of repositioned therapy for a monogenic disease
}

\author{
Eric J. Kort MD MS ${ }^{1,2}$, Nazish Sayed MD $\mathrm{PhD}^{3}$, Chun Liu $\mathrm{PhD}^{3}$, Sean M. Wu MD PhD ${ }^{3,4}$, \\ Joseph C. Wu MD $\mathrm{PhD}^{3,4,5}$, Stefan Jovinge $\mathrm{MD} \mathrm{PhD}^{1,3, *}$
}

\section{Affiliations:}

${ }^{1}$ DeVos Cardiovascular Research Program, Fredrik Meijer Heart and Vascular Institute, Spectrum Health \& Van Andel Institute, Grand Rapids, Michigan, USA.

${ }^{2}$ Department of Pediatrics \& Human Development, Michigan State University, Grand Rapids, Michigan, USA.

${ }^{3}$ Cardiovascular Institute, Stanford University, Stanford, CA USA

${ }^{4}$ Department of Medicine, Division of Cardiovascular Medicine, Stanford University, Stanford, CA, USA

${ }^{5}$ Department of Radiology, Stanford University, Stanford, CA USA

${ }^{*}$ Correspondence to Stefan Jovinge: Stefan.jovinge@,vai.org 


\begin{abstract}
The cost of drug development from initial concept to FDA approval has been estimated to be about 2.6 billion USD. ${ }^{1}$ This cost precludes development of targeted therapies for rare diseases such as monogenetic cardiomyopathies. As part of the Library of Integrated Network-based Cellular Signatures (LINCS) program funded by the NIH, the Broad Institute of MIT has publicly released transcriptional profiles quantifying the effects of more than 25,000 perturbagens on the expression of 978 genes in up to 77 cell lines. ${ }^{2}$ Transcriptomics has been shown to be a powerful tool in repurposing drugs $s^{3,4}$ and this dataset affords us the unique opportunity to systematically identify small molecule mimics or inhibitors of specific genes, thereby identifying novel treatments for genetic disorders. In this report, we take this approach to identify a novel drug therapy for a monogenic form of familial dilated cardiomyopathy with the transcriptional profile of FDA approved drugs. This approach could potentially be replicated for a wide range of monogenic diseases.
\end{abstract}

\title{
Background
}

Mutations in the LMNA gene are associated with familial dilated cardiomyopathy ${ }^{5-7}$ as well as premature aging syndromes. ${ }^{8}$ LMNA encodes for Lamin A/C, a nuclear structural protein which also plays a key role in chromatin organization and transcriptional regulation. ${ }^{9}$ These mutations may be either missense mutations, leading to severe early disease presumably due to dominant negative or pathological gain of function, or nonsense mutations characterized by late-onset disease due to haploinsufficiency. ${ }^{10,11}$ LMNA has been shown to be involved in the regulation of several signaling pathways whose disruption may contribute to the disease phenotype. These include PDGF signaling, autophagy via Akt/Beclin signaling, MAPK signaling, and histone modification. ${ }^{12-16}$ 


\section{Results}

In this study, we leveraged analysis of the LINCS L1000 dataset combined with iPSC based in vitro assays to identify FDA approved compounds that can reverse the effects of LMNA mutation at the transcriptional level (Fig. 1). We first obtained the Level 3 (normalized expression value) data from the NIH LINCS program. For our analysis, we utilized only the 978 directly measured genes (the "landmark" genes). The z-scores published by the LINCS program at the time we conducted this analysis were calculated for each sample vs. all the other samples on each experimental plate. For the purposes of our analysis, we wanted the z-scores calculated vs. controls only. Therefore, we calculated robust z-scores for each treated sample (

$\frac{x-\mu_{v_{1 / 2}}}{\operatorname{MAD} \times 1.482}$ where $\mu_{v_{1 / 2}}$ is the median value for the appropriate vehicle or empty vector controls on each plate).

We next sought to identify those genes that consistently and specifically shifted in expression as a result of LMNA knockdown (Fig. 2). To find genes that consistently responded to LMNA knockdown, we performed a "rank of ranks" analysis. First, we ranked the z-scores (genes) within each sample that was treated with LMNA-targeting short hairpins $(\mathrm{n}=84)$ constructs in the LINCS dataset (Fig. 2A, left-hand panel). Next, we combined these ranks across the samples into a single list and tested each gene to see how random their ranks were across samples by means of the Kolmogorov Smirnov statistic-essentially inverting the traditional Gene Set Enrichment Analysis ${ }^{17}$ to quantify the enrichment of each gene across a set of samples rather than a set of genes within each sample (Fig. 2A, right hand panel).

We also wanted to know how specific these gene expression changes were to LMNA knockdown vs. artefactual components of the experimental set up of the LINCS assay or non- 
specific cellular responses to genetic perturbation. Therefore, we repeated the above gene scoring using 100,000 random sets of gene-perturbed samples, with each set having the same number of samples as our LMNA perturbed set. This procedure allowed us to determine the probability that the differential expression statistic calculated for each gene from the LMNA perturbed samples was a unique feature of LMNA perturbation as opposed to a generic response frequently observed in random sets of gene-perturbed samples (Fig. 2b). The resulting bootstrapped $\mathrm{p}$-values were adjusted to control the false discovery rate (FDR). ${ }^{18}$

Interestingly, while the most extreme differential scores were all highly significant as determined by this analysis, there were several genes that had intermediate differential expression scores that were nevertheless highly significant when compared to random perturbations (Fig. 2B). We selected those genes with FDR adjusted p-value $<0.001$ and an enrichment $(\mathrm{KS})$ score $>0.2$ (absolute value) as our LMNA knockdown signature (supplemental Table S1). These genes were quite consistently down- or up-regulated across samples and cell lines (Fig. 2C).

PDGFA was among the most upregulated genes in our LMNA knockdown signature, consistent with recent work documenting activation of PDGF signaling in cardiomyocytes harboring mutation of LMNA. ${ }^{12}$ In addition, we performed Gene Ontology term enrichment analysis $^{19}$ on the Biological Function Ontology for the genes in our signature (Fig 2C). This analysis identified multiple GO terms related to autophagy, apoptosis, and hypoxia response that were enriched in our LMNA knockdown gene set, also consistent with prior work. ${ }^{7,20}$

We next sought to identify drugs that could reverse this LMNA knockdown signature. There is some evidence that parametric approaches are quantifiably superior to non-parametric scoring metrics for this type of gene signature enrichment analysis. As a result, we chose the 
XSUM statistic to quantify reversal of the LMNA signature within drug treated samples. ${ }^{21}$ Since there were many samples per drug in the L1000 dataset, we took the median score for each drug. In this way, we informatically screened over 600 FDA approved drugs for their ability to reverse the LMNA knockdown signature. We again assigned p-values to these scores by permutation (based on 10,000 random gene signatures).

As validation, we submitted our LMNA signature to the original CMAP enrichment tool (https://portals.broadinstitute.org/cmap/) $)^{22}$ to see what drugs could reverse our knockdown signature as determined by the CMAP connectivity score (Table S2). Of the FDA approved drugs in our L1000 dataset, 6 demonstrated a significant ability to reverse the LMNA signature according the CMAP enrichment tool. Of these 6 drugs, 4 also had significant enrichment scores based on the XSUM statistic derived from the L1000 dataset (Fig. 3A). These 4 drugs had stronger enrichment scores based on the original CMAP tool compared to the 2 drugs that were not enriched in the L1000 analysis.

We noted that multiple angiotensin receptor blockers (ARBs) exhibited significant reversal of the LMNA knockdown signature (Table S3 and Fig 4B), including olmesartan which was among the group of drugs that reversed this signature most significantly. However, not all ARBs exhibited this feature. It has previously been demonstrated that the various members of this drug class have variable transcriptional effects. ${ }^{23-25}$ When we analyzed all ARBs in our dataset as a group, this class of drugs collectively exhibited a significant reversal of the LMNA knockdown signature, but this effect was weaker for the entire class than for olmesartan or irbesartan specifically (FDR $=0.0147$ vs. 0.0001 and 0.0021 , respectively).

When we examined the effect of the highest scoring ARBs (olmesartan and irbesartan) on the expression of the individual genes in the LMNA knockdown signature, we noted that 
these drugs down regulated many of the genes upregulated by LMNA knockdown, and vice versa (Fig 4C). There was no such trend apparent for telmisartan, which did not exhibit significant enrichment in our analysis. However, the transcriptional effects of olmesartan and irbesartan were not universal with respect to the LMNA regulated genes, indicating that these drugs were acting on only portions of the LMNA knockdown "axis".

We next tested whether olmesartan and/or irbesartan treatment could influence the expression of cardiac markers and/or function of cardiomyocytes derived from induced pluripotent stem cells (iPSC-CMs) harboring a disease-related LMNA nonsense mutation. These cells harbor a heterozygous insertion of a guanine between nucleotides 348 and 349, causing a frameshift mutation at codon 117 , and resulting premature stop at codon 129. In iPSCCMs from a healthy control patient treated for 48 hours, a slight increase in MYH6 was observed in olmesartan treated cells, and a slight increase in TNNT2 expression was observed in captopril treated cells (Fig. 4A). In contrast, there was a 1.5-fold increase in MYH6, MYH7, and TNNT2 in olmesartan treated iPSC-CMs derived from a patient harboring a nonsense LMNA mutation. Other treatments tested either had no effect on these markers or suppressed their expression. We hypothesized that increased expression of these sarcomeric genes would correspond to improved contractility in these LMNA mutant cells.

To test that hypothesis, we examined what effect, if any, treatment of iPSC-CMs with these drugs had on the functional properties of these cells. For this, we examined the contractile properties of healthy control and LMNA-mutated iPSC-CMs. Irbesartan treatment of iPSCCMs derived from a control patient produced a very small (though statistically significant) increase in contraction velocity (Fig 4B), but no significant effect on contractile rate relative to DMSO treated controls, while olmesartan had no effect on the behavior of these cells relative 
to DMSO control. In contrast, olmesartan treatment of iPSC-CMs derived from the LMNAmutation patient resulted in a marked increase in both contraction velocity and beating rate. While there was a trend towards increased relaxation velocity in olmesartan treated cells compared to DMSO only control, this difference was not significant. However, olmesartan treatment was associated with significantly faster relaxation velocity as compared to the other drugs tested. These observations support the hypothesis that olmesartan will not exacerbate any underlying diastolic dysfunction and may in fact be favorable to other heart failure medications with respect to diastolic function.

\section{Conclusions}

These results demonstrate that the LINCS L1000 database can be exploited to perform an in silico screen for repositionable drugs that reverse the transcriptional consequences of specific genetic perturbations. One of two treatments we identified in such a way for LMNA mutation related cardiomyopathy showed a favorable response in our in vitro model-suggesting that this approach to drug repositioning for rare diseases may be a promising alternative to the traditional drug development pipeline.

\section{Methods}

\section{Statistics}

No statistical methods were used to predetermine sample size. The experiments were not randomized. The investigators who performed the phenotype assessment of the CMs were blinded to group allocation during experiments and data collection. The studies comply with all ethical regulations. 


\section{Data Availability}

This analysis was performed on the initial release for the LINCS L1000 dataset. Updated data from LINCS is available under accession \#GSE92742. The relevant same plate vehicle/vector control z-scores we calculated from the original LINCS L1000 data release, as well as R scripts to reproduce the analysis and figures presented here, are available at https:/github.com/vanandelinstitute/Lamin.

\section{LMNA Gene Signature Definition}

Full details of the generation of the LMNA gene expression signature and drug selection based on that signature using the LINCS L1000 database, are provided in a supplementary file (data_analysis.html). This information and supporting data required to repeat the analysis presented in this paper (including regenerating the figures) are freely available from github: https://github.com/vanandelinstitute/Lamin. Briefly, normalized gene expression data was obtained from the LINCS L1000 program. We then calculated the robust z-score for each gene within each sample relative to vehicle treated samples of the same cell type on the same 384 well plate. We extracted the z-scores for all instances treated with short hairpins targeting LMNA. This data was ranked sample-wise. Second, the entire matrix of ranks (978 genes by 84 shRNA samples) was ranked and Kolmogorov Smirnov analysis was performed on the position of each occurrence of each gene within this vector of ranks. The resulting analysis quantifies the extent to which the expression of each gene was consistently biased up or down relative to all other genes.

Finally, a bootstrapping procedure as performed to estimate significance of the KS score for each gene. We calculated KS scores for 100,000 random sets of shRNA treated samples (84 
samples in each set to match the LMNA set) in the LINCS data. The resulting p-values were adjusted for multiple comparisons using the method of Benjamini and Hochberg to control the false discovery rate at less than $5 \% .^{18}$

\section{Drug Selection}

To estimate the bias in gene expression for the genes in our LMNA signature within each sample in the L1000 dataset treated with and FDA approved compound, we used the XSUM metric because there is some evidence it is among the more performant algorithms for CMAP type data. ${ }^{21}$ The XSUM limits its search to the top N variable genes. However, since the L1000 dataset is already confined to the 978 most variant genes in the genome as determined by the LINCS program, we did not filter the gene set further. Therefore, we take the sum of the zscores for our upregulated genes and subtract the sum of the z-scores of the down regulated genes within the 978 L1000 genes for each drug perturbed instance. Because there are multiple instances per drug, we collapsed these scores to a single score per drug by taking the median.We again used a bootstrapping procedure to estimate the significance of each drug's score relative to random perturbations. We scored 10,000 random gene signatures (each with the same number of "up" and "down" regulated genes as the LMNA signature) to estimate how specific each drug was to the LMNA signature. Drugs were then ranked based on their bootstrapped p-value.

\section{Generation of human iPSCs}

Protocol for isolation and use of patient blood-derived peripheral blood mononuclear cell (PBMC) were approved by the Stanford University Human Subjects Research Institutional Review Board. PBMCs were isolated using a Ficoll-Paque PLUS gradient (GE Healthcare) and expanded as previously reported. ${ }^{26}$ For reprogramming, 1 million PBMCs were plated in 
medium supplemented with four OSKM reprogramming factors (CytoTune-iPS Sendai Reprogramming Kit, Life Technologies) according to the manufacturer's recommendations. The medium was changed after $24 \mathrm{hr}$ transfection and transferred to E7N medium (E8 medium minus TGF $\beta 1$ and $200 \mu \mathrm{M}$ sodium butyrate) on day 3. Colonies were picked into 1 well of a 12-well plate (1-colony in each well) on around day 20 and cultured in E8 with $10 \mu \mathrm{M}$ Y-27632 (Selleckchem). hiPSCs were then expanded into 6-well plated (coated with 1:200 growth factorreduced Matrigel) and maintained in E8 medium. Confluent hiPSCs were passaged every four days using 0.5 mM EDTA.

\section{Differentiation of hiPSCs to cardiomyocytes}

hiPSCs were routinely maintained in 6-well plates as described above. Cells were grown to reach $90 \%$ confluency and then subjected to differentiation in RPMI/B27 without insulin medium (Life Technologies) supplemented with $6 \mu$ M CHIR99021 (Selleckchem). Following $48 \mathrm{~h}$, the cells were subjected to the same medium supplemented with $4 \mu \mathrm{M}$ IWR-1-endo (Selleckchem). On day 7, the medium was changed to RPMI-B27 with insulin and exchanged every other day. Beating hiPSC-CMs usually can be observed around day 7 to day 10 . On day 11, the medium was switched to RPMI-B27 without D-glucose (Life Technologies) for 4 days to purify cardiomyocytes. For drug treatment and function analysis, purified iPSC-CMs were dissociated using TrypLE Express (Life Technologies) and re-plated to Matrigel-coated plates accordingly.

\section{Drug Treatment}

The indicated drug compounds were reconstituted from powder in DMSO to a working concentration of $10 \mathrm{mM}$. Drugs were then added to the cell culture wells to a final concentration 
of $10 \mu \mathrm{M}$ and the cell were returned to the cell culture incubator for the indicated times. Controls were treated with DMSO alone.

Quantitative real-time PCR.

RNA was extracted using a QIAGEN RNeasy kit following the manufacturer's instructions. cDNA was synthesized from 100 ng of total RNA using the High Capacity RNA-to-cDNA kit (ThermoFisher Scientific). Realtime-PCR was performed using TaqMan Gene Expression Master Mix and TaqMan probes (GAPDH, Hs02758991_g1; TNNT2, Hs00165960_m1; MYH6, Hs01101425_m1; MYH7, Hs01110632_m1). PCR reactions were conducted on 7900HT Real-Time PCR system (ThermoFisher Scientific) with triplicates and assessed using $\Delta \Delta \mathrm{Ct}$ relative quantification (RQ) method normalizing to GAPDH housekeeping gene.

High-content video-based cardiomyocyte contractility analysis

hiPSC differentiated cardiomyocytes were plated onto Matrigel-coated 96 well plates $(40,000$ per well) as described above. Following treatment with drugs, iPSC-CMs were examined on Sony SI8000 Live Cell Imaging System (Sony Biotechnology) with $\mathrm{CO}_{2}$ and $37{ }^{\circ} \mathrm{C}$ temperature incubation. Cell activities were recorded the beating video at a high frame rate (150 fps), focus and light conditions were automated controlled by the SI8000 software. After data acquisition, displacement and magnitudes of cardiomyocyte motions were calculated and presented using a motion detection algorithm by SI8000 software.

\section{Acknowledgement}

This work was mainly funded through Richard and Helen DeVos Foundation

\section{References}


1. Peters, S. \& Lowy, P. How the Tufts Center for the Study of Drug Development Pegged the Cost of a New Drug at $\$ 2.6$ Billion. (2014).

2. Subramanian, A., et al. A Next Generation Connectivity Map: L1000 Platform and the First 1,000,000 Profiles. Cell 171, 1437-1452 e1417 (2017).

3. Stegmaier, K., et al. Gene expression-based high-throughput screening(GE-HTS) and application to leukemia differentiation. Nat Genet 36, 257-263 (2004).

4. Stegmaier, K., et al. Gefitinib induces myeloid differentiation of acute myeloid leukemia. Blood 106, 2841-2848 (2005).

5. Fatkin, D., et al. Missense mutations in the rod domain of the lamin A/C gene as causes of dilated cardiomyopathy and conduction-system disease. $N$ Engl J Med 341, 1715-1724 (1999).

6. Pan, H., et al. A novel mutation in LAMIN A/C is associated with isolated early-onset atrial fibrillation and progressive atrioventricular block followed by cardiomyopathy and sudden cardiac death. Heart Rhythm 6, 707-710 (2009).

7. Siu, C.W., et al. Modeling of lamin A/C mutation premature cardiac aging using patientspecific induced pluripotent stem cells. Aging (Albany NY) 4, 803-822 (2012).

8. Ahmed, M.S., Ikram, S., Bibi, N. \& Mir, A. Hutchinson-Gilford Progeria Syndrome: A Premature Aging Disease. Mol Neurobiol 55, 4417-4427 (2018).

9. Butin-Israeli, V., Adam, S.A., Goldman, A.E. \& Goldman, R.D. Nuclear lamin functions and disease. Trends Genet 28, 464-471 (2012).

10. Perez-Serra, A., et al. A novel mutation in lamin a/c causing familial dilated cardiomyopathy associated with sudden cardiac death. J Card Fail 21, 217-225 (2015). 
11. Nishiuchi, S., et al. Gene-Based Risk Stratification for Cardiac Disorders in LMNA Mutation Carriers. Circ Cardiovasc Genet 10(2017).

12. Lee, J., et al. Activation of PDGF pathway links LMNA mutation to dilated cardiomyopathy. Nature (2019).

13. Choi, J.C., et al. Temsirolimus activates autophagy and ameliorates cardiomyopathy caused by lamin A/C gene mutation. Sci Transl Med 4, 144ra102 (2012).

14. Muchir, A., et al. Activation of MAPK pathways links LMNA mutations to cardiomyopathy in Emery-Dreifuss muscular dystrophy. J Clin Invest 117, 1282-1293 (2007).

15. Galiova, G., Bartova, E., Raska, I., Krejci, J. \& Kozubek, S. Chromatin changes induced by lamin A/C deficiency and the histone deacetylase inhibitor trichostatin A. Eur J Cell Biol 87, 291-303 (2008).

16. Mattioli, E., et al. Statins and Histone Deacetylase Inhibitors Affect Lamin A/C - Histone Deacetylase 2 Interaction in Human Cells. Front Cell Dev Biol 7, 6 (2019).

17. Subramanian, A., et al. Gene set enrichment analysis: a knowledge-based approach for interpreting genome-wide expression profiles. Proc Natl Acad Sci US A 102, 15545-15550 (2005).

18. Benjamini, Y. \& Hochberg, Y. Controlling the false discovery rate: a practical and powerful approach to multiple testing. J Roy Statist Soc Ser B 57, 1 (1995).

19. Yu, G., Wang, L.G., Han, Y. \& He, Q.Y. clusterProfiler: an R package for comparing biological themes among gene clusters. OMICS 16, 284-287 (2012).

20. Chiarini, F., et al. The Cutting Edge: The Role of mTOR Signaling in Laminopathies. Int J Mol Sci 20(2019). 
21. Cheng, J., et al. Evaluation of analytical methods for connectivity map data. Pac Symp Biocomput, 5-16 (2013).

22. Lamb, J., et al. The Connectivity Map: using gene-expression signatures to connect small molecules, genes, and disease. Science 313, 1929-1935 (2006).

23. Benson, S.C., et al. Identification of telmisartan as a unique angiotensin II receptor antagonist with selective PPARgamma-modulating activity. Hypertension 43, 993-1002 (2004).

24. Yuen, C.Y., et al. Telmisartan inhibits vasoconstriction via PPARgamma-dependent expression and activation of endothelial nitric oxide synthase. Cardiovasc Res 90, 122-129 (2011).

25. Kurtz, T.W. \& Pravenec, M. Molecule-specific effects of angiotensin II-receptor blockers independent of the renin-angiotensin system. American journal of hypertension 21, 852859 (2008).

26. Burridge, P.W., et al. Chemically defined generation of human cardiomyocytes. Nature methods 11, 855-860 (2014). 


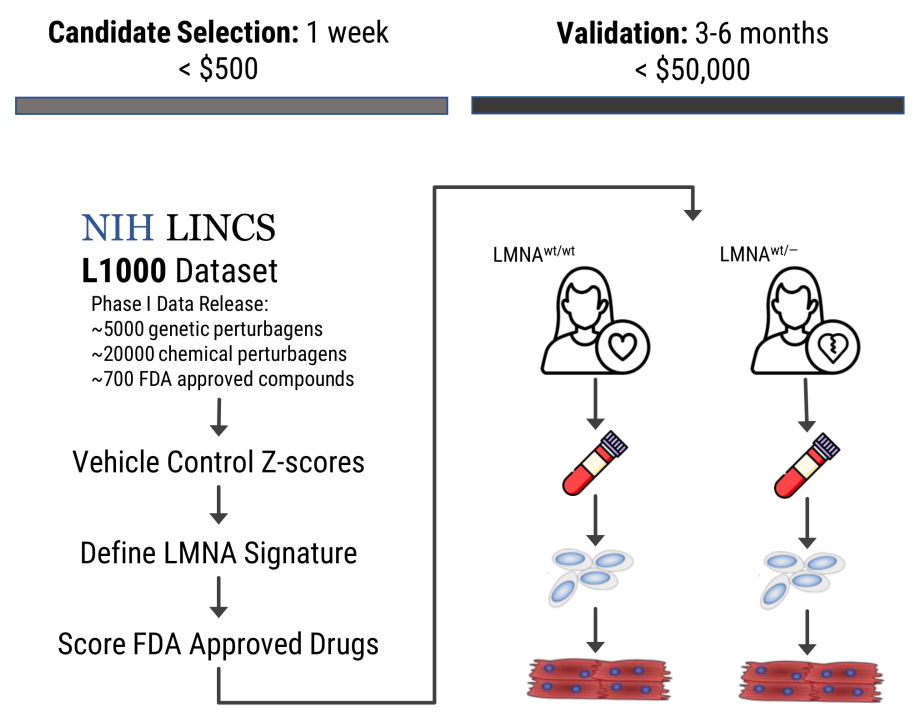

Figure 1. Overview of approach. The LINCS L1000 data was downloaded, and expression levels were converted to z-scores by comparing each treated sample to corresponding controls. From this dataset, we identify a gene-specific signature (in this case, the signature describing the transcriptional consequences of LMNA knockdown). With this signature, gene-targeting drugs are identified. The candidate drugs are then validated - in this case by testing in patient and healthy control iPSC-derived cardiomyocytes. Estimated duration and cost of this repositioning pipeline are provided for reference. 
A
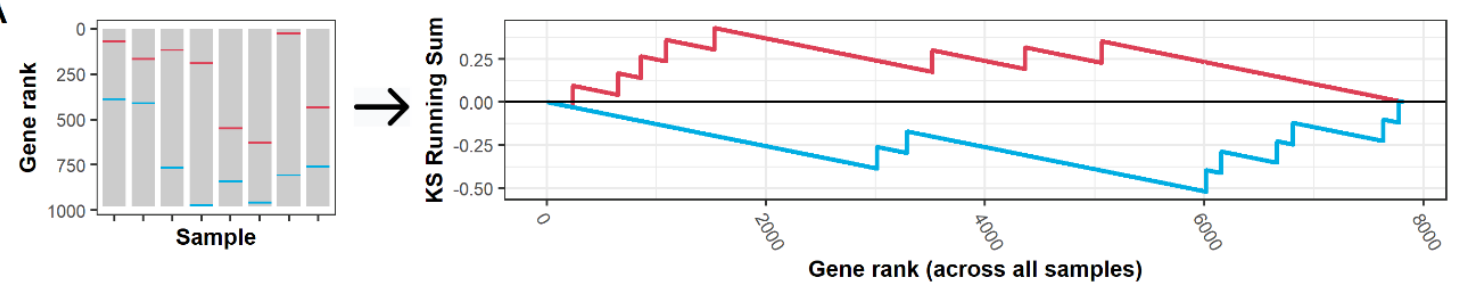

Gene

= RHOA

B

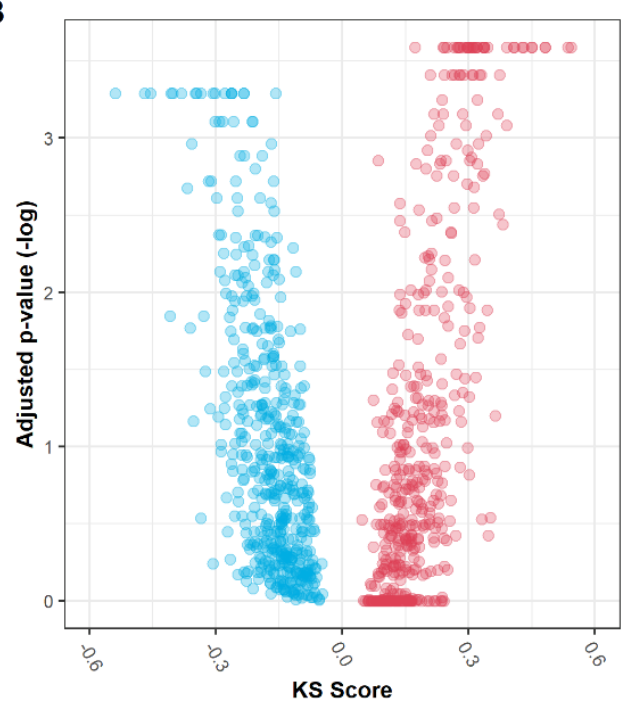

C

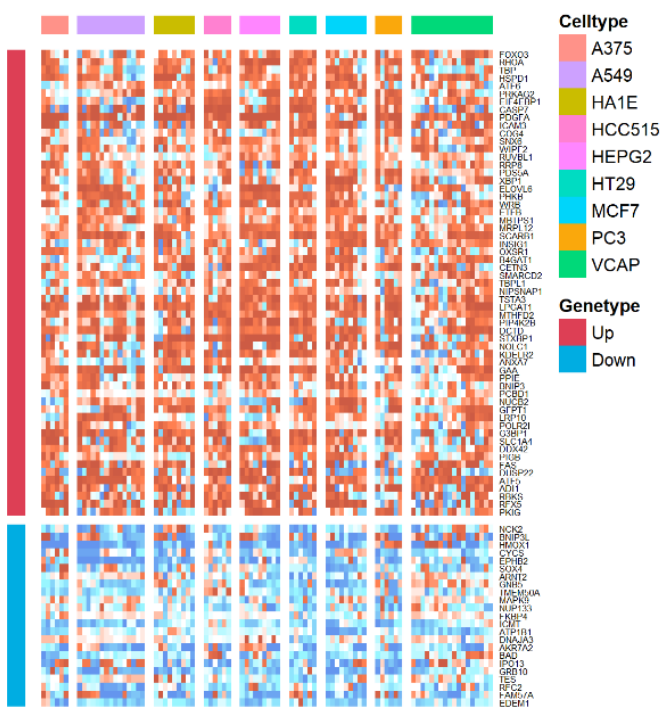

D


Figure 2. Establishment of LMNA knockdown gene signature. (A) Genes were scored based on how consistently and how strongly they were up- or down-regulated by LMNA knockdown in the LINCS L1000 database. Permutation of random sets of short hairpin perturbed samples was used to estimate the significance of these expression shifts. We selected those genes with both a KS score $>0.3$ and $a-\log$ p-value $>0.3$ as our LMNA knockdown gene set. (B) The LMNA knockdown gene signature showed fairly uniform up- or down-regulation across all samples in the L1000 dataset across all cell types treated. (C) Gene ontology enrichment analysis suggests that the genes in this signature are involved in pathways related to autophagy and apoptosis. 
A

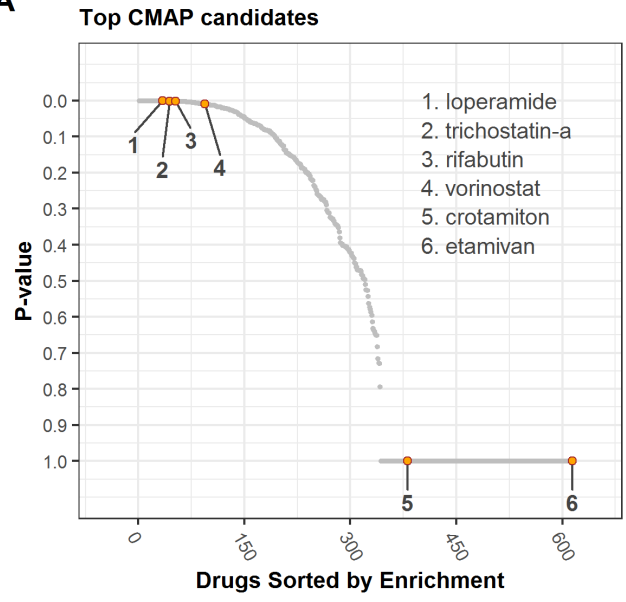

B

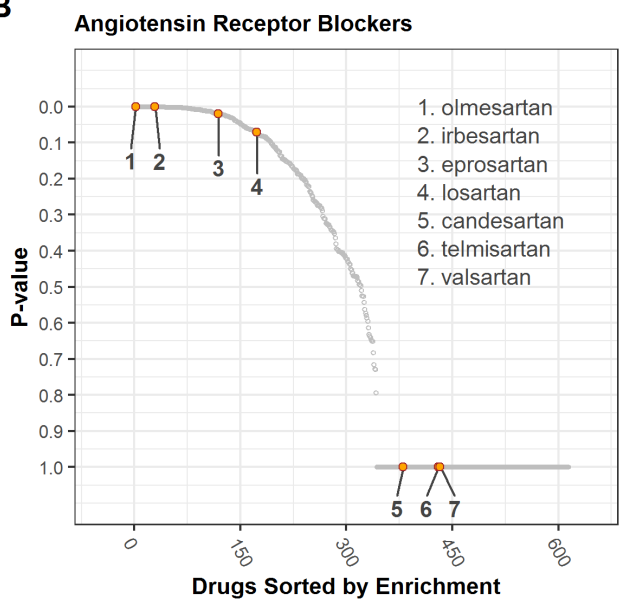

C

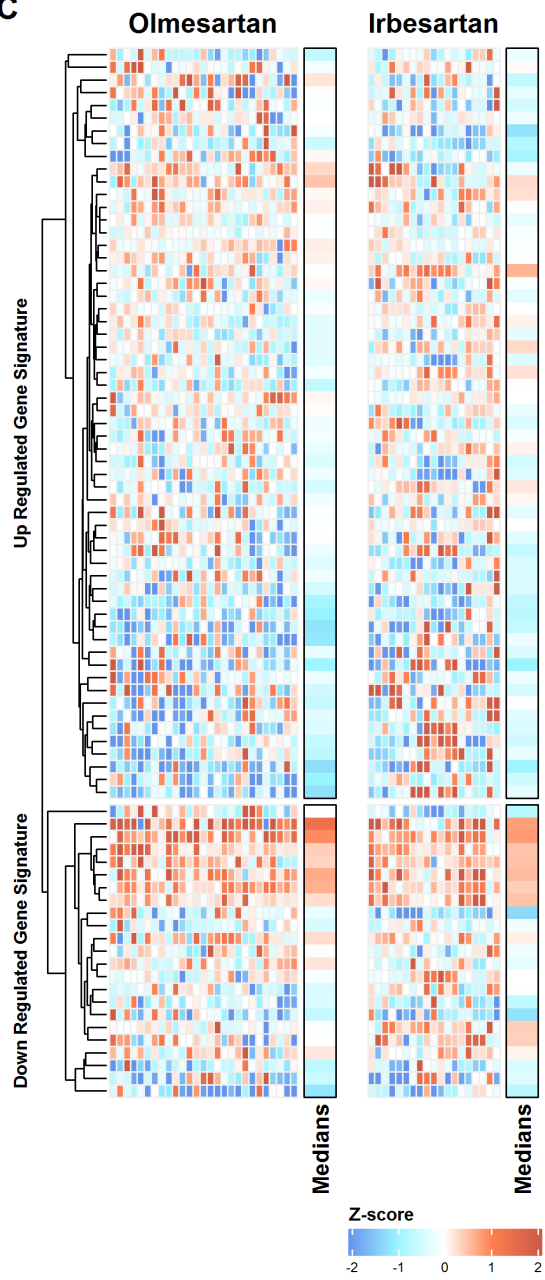

Telmisartan



Figure 3. The LMNA knockdown signature was used to identify drugs that could reverse these transcriptional changes in vitro. (A) We first submitted the gene signature to the original CMAP web tool. Of the top 6 drugs that most strongly reversed the LMNA knockdown signature based on that tool, 4 also exhibited highly significant reversal of this signature in the LINCS L1000 data, suggesting there was some coherence between the various platforms and analytic approaches used by these systems. (B) We note that two angiotensin receptor blockers were among the most highly significant drugs based on their ability to reverse the LMNA knockdown signature. Indeed, 3 of 7 ARBs in the database showed gene set enrichment with an FDR adjusted p-value of $<0.05$. (C) These ARBs do not reverse the entirety of the LMNA signature. Rather, high scoring ARBs (olmesartan and irbesartan) reverse highly overlapping segments of the LMNA signature, whereas telmisartan (a low scoring drug) exhibits a more random expression pattern for the genes in the LMNA knockdown signature. 
A

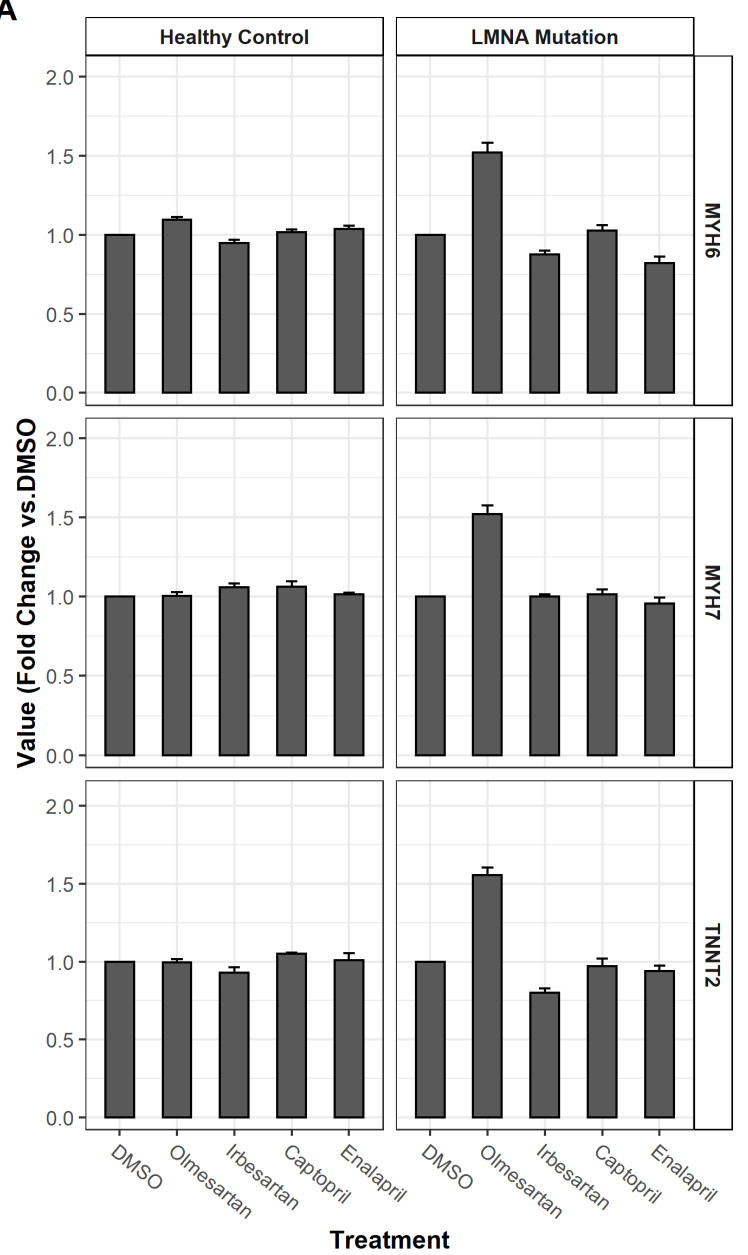

B
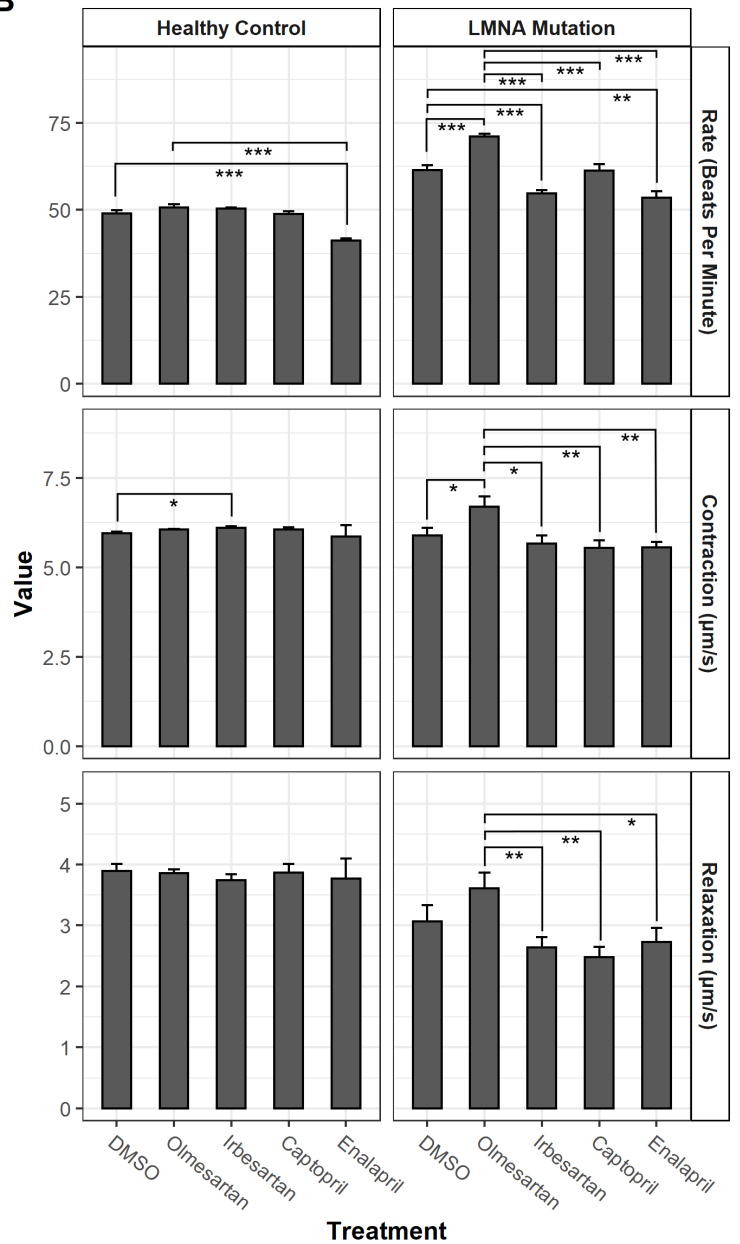

Figure 4. In vitro validation of the effect of predicted drugs on cardiomyocyte transcription and phenotype. All results are from duplicate experiments, with 3 technical replicates per experiment (total $\mathrm{N}=6$ ). All error bars are +SEM. (A) We measured the expression of three cardiac markers (MYH6, MYH7, and TNNT2) by quantitative PCR in cardiomyocytes derived from iPSC cells from a healthy control and a patient harboring a LMNA mutation. Fold-change relative to DMSO treated controls was calculated for each marker after 48 hours of treatment with the drugs shown, which include 2 of the ARBs identified by our analysis, and two angiotensin converting enzyme inhibitors used as controls due to their canonical activity on the same pathway as ARBs. Significant differences from DMSO treated samples are indicated $(*: \mathrm{p} \leq 0.05, * *: \mathrm{p} \leq 0.01, * * *: \mathrm{p} \leq 0.001$ by two-tailed t-tests). (B) Beating rate, contractile velocity, and relaxation velocity were measured by quantitative video microscopy in both control and LMNA mutant cardiomyocytes. Rate is expressed in beats per minute. Contractile force is expressed in micrometers per second. Significant differences from DMSO and/or olmesartan treated samples are indicated $(*: \mathrm{p} \leq 0.05$, $*^{*}: \mathrm{p} \leq 0.01, * * *: \mathrm{p} \leq 0.001$ by two-tailed t-tests). 


\begin{tabular}{|c|c|c|c|c|c|}
\hline L1000 id & Entrez ID & Symbol & Direction & Score & Adj. P \\
\hline 204131_s_at & 2309 & FOXO3 & up & 0.31038 & 0.00026 \\
\hline 200059_s_at & 387 & RHOA & up & 0.33561 & 0.00026 \\
\hline 200807_s_at & 3329 & HSPD1 & up & 0.45058 & 0.00026 \\
\hline $203952^{-}$at & 22926 & ATF6 & up & 0.31712 & 0.00026 \\
\hline 207181_s_at & 840 & CASP7 & up & 0.34447 & 0.00026 \\
\hline 205463_s_at & 5154 & PDGFA & up & 0.60383 & 0.00026 \\
\hline 204949_at & 3385 & ICAM3 & up & 0.33742 & 0.00026 \\
\hline 212189_s_at & 25839 & COG4 & up & 0.23888 & 0.00026 \\
\hline 217789_at & 58533 & SNX6 & up & 0.29959 & 0.00026 \\
\hline 203171_s_at & 23378 & RRP8 & up & 0.29550 & 0.00026 \\
\hline 200670_at & 7494 & XBP1 & up & 0.27354 & 0.00026 \\
\hline 202749_at & 7485 & WRB & up & 0.33783 & 0.00026 \\
\hline 202942_at & 2109 & ETFB & up & 0.31963 & 0.00026 \\
\hline 201620_at & 8720 & MBTPS1 & up & 0.31108 & 0.00026 \\
\hline 203931_s_at & 6182 & MRPL12 & up & 0.39031 & 0.00026 \\
\hline 201819_at & 949 & SCARB1 & up & 0.54351 & 0.00026 \\
\hline 201827_at & 6603 & SMARCD2 & up & 0.29545 & 0.00026 \\
\hline 208398_s_at & 9519 & TBPL1 & up & 0.26473 & 0.00026 \\
\hline 36936_at & 7264 & TSTA3 & up & 0.42983 & 0.00026 \\
\hline 202651_at & 9926 & LPGAT1 & up & 0.53546 & 0.00026 \\
\hline 201761_at & 10797 & MTHFD2 & up & 0.40639 & 0.00026 \\
\hline 201572_x_at & 1635 & DCTD & up & 0.44987 & 0.00026 \\
\hline 200698_at & 11014 & KDELR2 & up & 0.27340 & 0.00026 \\
\hline 202812_at & 2548 & GAA & up & 0.48100 & 0.00026 \\
\hline 202494_at & 10450 & PPIE & up & 0.30327 & 0.00026 \\
\hline 201849_at & 664 & BNIP3 & up & 0.27682 & 0.00026 \\
\hline 203675_at & 4925 & NUCB2 & up & 0.24168 & 0.00026 \\
\hline 202722_s_at & 2673 & GFPT1 & up & 0.30721 & 0.00026 \\
\hline $201412^{-}$at & 26020 & LRP10 & up & 0.24893 & 0.00026 \\
\hline 201503_at & 10146 & G3BP1 & up & 0.48116 & 0.00026 \\
\hline 212811_x_at & 6509 & SLC1A4 & up & 0.27994 & 0.00026 \\
\hline 201788_at & 11325 & DDX42 & up & 0.33371 & 0.00026 \\
\hline 204781_s_at & 355 & FAS & up & 0.29861 & 0.00026 \\
\hline 218845_at & 56940 & DUSP22 & up & 0.31922 & 0.00026 \\
\hline $204999^{-} \mathrm{s}$ at & 22809 & ATF5 & up & 0.42710 & 0.00026 \\
\hline 217761_at & 55256 & ADI1 & up & 0.40836 & 0.00026 \\
\hline 202732_at & 11142 & PKIG & up & 0.28803 & 0.00026 \\
\hline 221539_at & 1978 & EIF4EBP1 & up & 0.26660 & 0.00039 \\
\hline 212051_at & 147179 & WIPF2 & up & 0.28797 & 0.00039 \\
\hline 201614_s_at & 8607 & RUVBL1 & up & 0.30746 & 0.00039 \\
\hline 204256_at & 79071 & ELOVL6 & up & 0.32921 & 0.00039 \\
\hline 202738_s_at & 5257 & PHKB & up & 0.27767 & 0.00039 \\
\hline 203188_at & 11041 & B4GAT1 & up & 0.26010 & 0.00039 \\
\hline 201709_s_at & 8508 & NIPSNAP1 & up & 0.20795 & 0.00039 \\
\hline 201080_at & 8396 & PIP4K2B & up & 0.31016 & 0.00039 \\
\hline 202260_s_at & 6812 & STXBP1 & up & 0.37298 & 0.00039 \\
\hline 212955_s_at & 5438 & POLR2I & up & 0.32508 & 0.00039 \\
\hline 205452_at & 9488 & PIGB & up & 0.24231 & 0.00039 \\
\hline 219222_at & 64080 & RBKS & up & 0.27915 & 0.00039 \\
\hline
\end{tabular}




\begin{tabular}{llllll} 
L1000 id & Entrez ID & Symbol & Direction & Score & Adj. P \\
\hline 207842_sat & NA & NA & up & 0.23652 & 0.00056 \\
203557_saat & 5092 & PCBD1 & up & 0.32056 & 0.00056 \\
218292_sat & 51422 & PRKAG2 & up & 0.23998 & 0.00070 \\
212140_at & 23244 & PDS5A & up & 0.28592 & 0.00070 \\
205895_saat & 9221 & NOLC1 & up & 0.36963 & 0.00070 \\
209860_saat & 310 & ANXA7 & up & 0.21816 & 0.00070 \\
203135_at & 6908 & TBP & up & 0.22961 & 0.00083 \\
201626_at & 3638 & INSIG1 & up & 0.29395 & 0.00083 \\
202963_at & 5993 & RFX5 & up & 0.39054 & 0.00083 \\
202696_at & 9943 & OXSR1 & up & 0.21009 & 0.00096 \\
209662_at & 1070 & CETN3 & up & 0.34161 & 0.00096 \\
221478_at & 665 & BNIP3L & down & 0.30824 & 0.00051 \\
203665_at & 3162 & HMOX1 & down & 0.53932 & 0.00051 \\
208905_at & 54205 & CYCS & down & 0.27988 & 0.00051 \\
209588_at & 2048 & EPHB2 & down & 0.45589 & 0.00051 \\
201416_at & 6659 & SOX4 & down & 0.26452 & 0.00051 \\
202986_at & 9915 & ARNT2 & down & 0.23398 & 0.00051 \\
204000_at & 10681 & GNB5 & down & 0.23472 & 0.00051 \\
201611_sat & 23463 & ICMT & down & 0.40633 & 0.00051 \\
201243_s_at & 481 & ATP1B1 & down & 0.38234 & 0.00051 \\
205963_s_at & 9093 & DNAJA3 & down & 0.26364 & 0.00051 \\
214259_s_at & 8574 & AKR7A2 & down & 0.30203 & 0.00051 \\
209364_at & 572 & BAD & down & 0.33628 & 0.00051 \\
203546_at & 9670 & IPO13 & down & 0.26234 & 0.00051 \\
209409_at & 2887 & GRB10 & down & 0.40340 & 0.00051 \\
203696_sat & 5982 & RFC2 & down & 0.34627 & 0.00051 \\
218898_at & 79850 & FAM57A & down & 0.34849 & 0.00051 \\
203279_at & 9695 & EDEM1 & down & 0.47046 & 0.00051 \\
203315_at & 8440 & NCK2 & down & 0.30229 & 0.00078 \\
217766_saat & 23585 & TMEM50A & down & 0.29173 & 0.00078 \\
203218_at & 5601 & MAPK9 & down & 0.21427 & 0.00078 \\
202184_saat & 55746 & NUP133 & down & 0.25876 & 0.00078 \\
200895_sat & 2288 & FKBP4 & down & 0.28478 & 0.00078 \\
202720_at & 26136 & TES & down & 0.21366 & 0.00078
\end{tabular}

Supplemental Table S1: LMNA Knockdown Signature Genes. Genes identified as consistently and specifically perturbed by LMNA targeting short hairpin treatment. The direction column indicates whether the gene is up-regulated or down-regulated by short hairpin treatment relative to controls. 


\begin{tabular}{cccccc}
\hline rank & cmap name & mean & n & enrichment & p \\
\hline 1 & vorinostat & -0.599 & 12 & -0.771 & 0 \\
2 & trichostatin A & -0.412 & 182 & -0.5 & 0 \\
3 & scriptaid & -0.684 & 3 & -0.941 & 0.00032 \\
4 & rifabutin & -0.608 & 3 & -0.889 & 0.00268 \\
5 & rolitetracycline & -0.247 & 4 & -0.801 & 0.00304 \\
7 & difenidol & -0.381 & 3 & -0.859 & 0.00563 \\
9 & methacholine & & & & \\
13 & chloride & -0.407 & 3 & -0.851 & 0.00659 \\
15 & levamisole & -0.325 & 4 & -0.728 & 0.01112 \\
16 & loperamide & -0.438 & 6 & -0.603 & 0.01357 \\
18 & sulfadiazine & -0.322 & 5 & -0.645 & 0.01402 \\
19 & lobelanidine & -0.35 & 4 & -0.706 & 0.01554 \\
20 & GW-8510 & -0.445 & 4 & -0.701 & 0.01649 \\
21 & calmidazolium & -0.542 & 2 & -0.906 & 0.01795 \\
23 & ticarcillin & -0.487 & 3 & -0.775 & 0.02342 \\
24 & MK-886 & -0.483 & 2 & -0.882 & 0.02813 \\
27 & imipenem & -0.358 & 4 & -0.665 & 0.02847 \\
29 & suloctidil & -0.339 & 4 & -0.651 & 0.03495 \\
31 & crotamiton & -0.43 & 4 & -0.644 & 0.03847 \\
33 & piracetam & -0.335 & 4 & -0.636 & 0.04269 \\
\hline
\end{tabular}

Supplemental Table S2: Drugs which significantly reverse the LMNA knockdown signature as determined by the original CMAP web tool. 


\begin{tabular}{|c|c|c|c|c|c|}
\hline Drug & P-value & Drug & P-value & Drug & P-value \\
\hline Acyclovir & 0.000100 & Erlotinib & 0.002700 & Ganciclovir & 0.012899 \\
\hline Chloramphenicol & 0.000100 & Prazosin & 0.002900 & Doxorubicin & 0.013599 \\
\hline Diclofenac & 0.000100 & Carteolol & 0.003100 & Paclitaxel & 0.013599 \\
\hline Elvitegravir & 0.000100 & Acetylcholine & 0.003200 & Citalopram & 0.013899 \\
\hline Imatinib & 0.000100 & Glimepiride & 0.003200 & Mitoxantrone & 0.015098 \\
\hline Olmesartan & 0.000100 & Alprazolam & 0.003400 & Methotrexate & 0.015198 \\
\hline Praziquantel & 0.000100 & Everolimus & 0.003400 & Entecavir & 0.016898 \\
\hline Carbidopa & 0.000200 & Benzonatate & 0.003600 & Ruxolitinib & 0.016998 \\
\hline Clopidogrel & 0.000200 & Methoxsalen & 0.003600 & Raltegravir & 0.018098 \\
\hline Isotretinoin & 0.000200 & Indinavir & 0.003800 & Nortriptyline & 0.018198 \\
\hline Ketoprofen & 0.000200 & Modafinil & 0.003800 & Meloxicam & 0.018498 \\
\hline Mafenide & 0.000200 & Plerixafor & 0.004000 & Vorinostat & 0.019498 \\
\hline Auranofin & 0.000300 & Levetiracetam & 0.004700 & Isosorbide & 0.019998 \\
\hline Minoxidil & 0.000300 & Disulfiram & 0.004900 & Daunorubicin & 0.020698 \\
\hline Olopatadine & 0.000300 & Trichostatin-A & 0.004900 & Gemcitabine & 0.021698 \\
\hline Tenofovir & 0.000300 & Prednisone & 0.005099 & Hydroflumethiazide & 0.021798 \\
\hline Dinoprostone & 0.000500 & Ingenol & 0.005199 & Droperidol & 0.022298 \\
\hline Homatropine & 0.000500 & Ranolazine & 0.005199 & Nicardipine & 0.022998 \\
\hline Metaxalone & 0.000600 & Cilastatin & 0.005299 & Nimodipine & 0.023198 \\
\hline Riluzole & 0.000600 & Penicillin & 0.005399 & Etoposide & 0.023898 \\
\hline Cefuroxime & 0.000700 & Gatifloxacin & 0.005999 & Cyclophosphamide & 0.024098 \\
\hline Fludarabine & 0.000700 & Panobinostat & 0.006099 & Fenofibrate & 0.025397 \\
\hline Fluvoxamine & 0.000700 & Ciclopirox & 0.006399 & Testosterone & 0.025397 \\
\hline Belinostat & 0.000800 & Edrophonium & 0.006999 & Tolmetin & 0.026097 \\
\hline Ramipril & 0.000800 & Etodolac & 0.007099 & Pimozide & 0.028097 \\
\hline Propafenone & 0.000900 & Amantadine & 0.007199 & Eprosartan & 0.028497 \\
\hline Temsirolimus & 0.001000 & Alitretinoin & 0.007299 & Epirubicin & 0.028997 \\
\hline Loperamide & 0.001100 & Nefazodone & 0.007399 & Pirfenidone & 0.028997 \\
\hline Cefotaxime & 0.001200 & Atenolol & 0.007599 & Brinzolamide & 0.030497 \\
\hline Pitavastatin & 0.001300 & Formoterol & 0.007599 & Doxylamine & 0.031697 \\
\hline Anagrelide & 0.001400 & Capecitabine & 0.008099 & Primaquine & 0.032197 \\
\hline Clozapine & 0.001400 & Amiodarone & 0.008199 & Propylthiouracil & 0.033097 \\
\hline Ponatinib & 0.001400 & Ezetimibe & 0.008199 & Fluconazole & 0.034397 \\
\hline Acamprosate & 0.001500 & Nafcillin & 0.008199 & Tretinoin & 0.035296 \\
\hline Rifabutin & 0.001600 & Topotecan & 0.008199 & Moxifloxacin & 0.036496 \\
\hline Diazoxide & 0.001700 & Balsalazide & 0.008599 & Flurbiprofen & 0.037596 \\
\hline Sildenafil & 0.001700 & Acarbose & 0.008799 & Ethosuximide & 0.038396 \\
\hline Imiquimod & 0.002000 & Cortisone & 0.008899 & Dactinomycin & 0.040596 \\
\hline Irbesartan & 0.002100 & Nizatidine & 0.008999 & Levofloxacin & 0.041196 \\
\hline Bumetanide & 0.002200 & Granisetron & 0.009199 & Ticlopidine & 0.041596 \\
\hline Isradipine & 0.002200 & Propofol & 0.009899 & Leflunomide & 0.042796 \\
\hline Busulfan & 0.002300 & Levocetirizine & 0.010099 & Ketorolac & 0.042896 \\
\hline Bimatoprost & 0.002400 & Vincristine & 0.010699 & Niacin & 0.046795 \\
\hline Venlafaxine & 0.002400 & Gefitinib & 0.010799 & Bortezomib & 0.047095 \\
\hline Quinine & 0.002600 & Metronidazole & 0.010799 & Fluocinolone & 0.048895 \\
\hline Carbamazepine & 0.002700 & Bethanechol & 0.011799 & & \\
\hline
\end{tabular}

Supplemental Table S3: Drugs which significantly reversed LMNA knockdown signature in our analysis of L1000 dataset. 